\title{
Effect of consecutive aerobic and resistance exercise on cortisol, immunoglobulin A, and creatine kinase responses in male students
}

\author{
Hamid Arazi ${ }^{1}$, Mohammad Azizi ${ }^{2}$ \\ 1Faculty of Physical Education and Sport Science, University of Guilan, Rasht, ${ }^{2}$ Faculty of Physical Education \\ and Sport Science, University of Razi- Kermanshah, Iran
}

\section{Summary}

Study aim: To investigate the effects of consecutive aerobic and resistance exercise on Cortisol, Immunoglobulin A (IgA), and Creatine kinase (CK) responses of male students.

Material and methods: Eight subjects (mean age $21.0 \pm 1.5$ years) completed two trail, aerobic ergometer exercises (60\% VO2max; 45-min) and resistance (80\% 1RM; 45-min) exercise concurrently; each subject was his own control. Blood samples were collected pre-exercise, post-exercise, and 3 hours after exercise. One-way ANOVA with repeated measure and LSD post-hoc tests were used to evaluate changes in Cortisol, IgA, and CK during baseline and exercise periods.

Results: During baseline, there were no differences between exercise day (ED) values and those obtained at rest day (RD) for Cortisol, IgA, and CK. After exercise, Cortisol concentration in $\mathrm{ED}$ was significantly higher than $\mathrm{RD}(\mathrm{p}<0.05)$; however, changes in IgA and CK responses were not significant.

Conclusions: This type of consecutive exercise didn't increase susceptibility to upper respiratory tract infection and muscle damage. Therefore, it can be useful for the preparation phase of training for athletes.

Key words: Immunoglobulin - Mucosal immune - Stress hormone - Consecutive exercises

\section{Introduction}

The immune system is influenced by physiological and psychological stressors [12], including physical activity. The effects of exercise on the immune system are mediated through interactions between the nervous, endocrine, and immune systems [35].

Salivary immunoglobulin A (IgA) provides a valuable defense against potential pathogens by preventing colonization and replication on the mucosal surfaces of the upper respiratory tract [22]. Secretory $\operatorname{IgA}$ as an important component of saliva is produced in local plasma cells. The secreted IgA prevents antigens and microbes from adhering to and penetrating the epithelium (immune exclusion), interrupts replication of intracellular pathogens during transcytosis through epithelial cells (intracellular neutralization), and binds antigens in the lamina propria, thus facilitating their excretion through the epithelium back into the lumen (immune excretion) [11,19]. Reduced concentrations or reduction in the release of Salivary IgA may allow for increased pathogenesis via the mucosal surface [34]. It has been shown that lower concentrations of IgA or chronic IgA deficiencies are associated with an increased frequency of upper respiratory tract infection (URTI) episodes [10,11], recurrent URTI [15], or reduced protection against certain infections [2].

There are reports demonstrating that IgA concentrations are suppressed in response to high intensity exercise $[10,39]$ remain either unaltered $[26,42]$ or are elevated in response to moderate-low intensity exercise [20].

However, several studies have also shown a stable secretion rate of IgA following tennis drills [31], a soccer match [5], or cycling [6]. Jemmott and McClelland [16] concluded from a meta-analysis of nine studies that the level of $\operatorname{IgA}$ secretion might indicate vulnerability toward URTI. Mackinnon and Hooper [24] further suggested that the protective effect might depend not only on IgA concentration, but also on salivary flow rate.

Although the physiological mechanisms underlying the temporary suppression of various aspects of the immune function after high-intensity exercise are still unclear, it is likely that both neural and endocrine factors influence the immune response to exercise $[8,35]$. Cortisol is the end product of the neuroendocrine stress response in humans. Although high levels of Cortisol have been demonstrated to inhibit antibody production in vitro [1], 
studies have suggested that elevated glucocorticoids are necessary, but not sufficient, to suppress the antibody response [8]. However, while some studies have reported no acute changes in cortisol secretion in response to resistance exercise [7,29], others have reported changes [36].

Creatine kinase $(\mathrm{CK})$ is found predominantly in muscle and is released into circulation during muscular lesions. Therefore, serum CK activity has been theoretically expected to be useful as a marker in exercise physiology and sports medicine for the detection of muscle injury and overwork [13]. However, previous studies on CK release have not clearly demonstrated its value as a marker for these states [27]. Numerous studies have evaluated changes in CK activity after exercise and found that it differs markedly according to exercise conditions. For example, in isometric muscle contraction exercise, peak serum CK activity is observed relatively early, 24-48 h after exercise [18], whereas it is seen 3-7 days after exercise in eccentric muscle contraction exercise [27], and a biphasic pattern is observed in weight training [40].

Totsuka et al. [25] reported that CK response depends on individual fitness level. They show that peak $\mathrm{CK}$ values during the bicycle exercise correlated with workload/crosssectional area of the quadriceps femoris muscle, workload/volume of the quadriceps femoris muscle, and knee extensor strength/body mass.

However, according to inconsistency in previous results, there are no quantitative results concerning immune response to exercise. To our knowledge there is no study that evaluates the effect of single bout of consecutive aerobic and resistance exercise on immune response. Therefore, the purpose of this study was to determine the effect of a single bout of consecutive aerobic and resistance exercise on Cortisol, IgA, and CK responses in male students.

\section{Material and Methods}

Participants: Eight male physical education students took part in the study. They were not specifically endurance or resistance trained but were actively involved in various kinds of sports for 6-8 hours weekly on average. Characteristics of the subjects are reported in Table 1. Before the start of the study, subjects provided written informed consent. They were asked not to perform any strenuous exercise or consume medication for 2 days before each trial. Each subject was his own control. The protocol was approved by the Guilan University Ethics Committee.

Preliminary measurements: Maximal aerobic capacity $\left(\dot{\mathrm{VO}}_{2} \max \right)$ was estimated using the Fox protocol (heart rate response to $5 \mathrm{~min}$ of cycle ergometry at $150 \mathrm{~W}$ $\left(6300-19.26 \times(\mathrm{HR} 5 \mathrm{~min})=\dot{\mathrm{V}}{ }_{2} \mathrm{max}\right) ;$ [9]). Also, muscular strength was measured at the beginning of the study.
Strength was assessed by one repetition maximum (1-RM) for bench press, leg press, leg extension, leg curl, lats pull-down, and biceps curl.

Table 1. Characteristics of subjects (mean \pm SD)

\begin{tabular}{|c|c|}
\hline Variable & $\begin{array}{l}\text { Male } \\
\text { students }(n=8)\end{array}$ \\
\hline Age (years) & $21.0 \pm 1.51$ \\
\hline Body height (cm) & $178.0 \pm 5.0$ \\
\hline Body mass (kg) & $74.0 \pm 8.8$ \\
\hline Body fat (\%) & $12.3 \pm 3.6$ \\
\hline BMI $\left(\mathrm{kg} / \mathrm{m}^{2}\right)$ & $23.3 \pm 1.0$ \\
\hline$\dot{\mathrm{V}} \mathrm{O}_{2} \max (\mathrm{ml} / \mathrm{kg} / \mathrm{min})$ & $46.2 \pm 6.7$ \\
\hline
\end{tabular}

Following warm-up for the 6 resistance exercise testing, subjects selected a weight with which they felt they could complete three repetitions. At this weight, they only performed one repetition. Subjects then selected a weight they felt would be their 1-RM and attempted one repetition with this weight. Following successful attempts, weight was increased by $2-5 \mathrm{~kg}$ for subsequent $1-\mathrm{RM}$ attempts. The 1-RM was usually reached in less than 6 sets, including the warm-up set. There was 3 min of rest between attempts, and two assistants changed the weight on the bar between attempts.

Procedures: The subjects completed trials in a counterbalanced order. For the afternoon exercise trial, subjects reported to the laboratory at 14:00 after fasting from 22:30 the previous day to 10:30 a.m. They then performed 45 min cycling at $60 \% \mathrm{~V}_{2} \max$ and 45 min 6 free-weight resistance exercise in the following order: bench press, leg press, leg extension, leg curl, lats pull-down, and biceps curl. Exercises consisted of 3 sets of 8 repetitions (80\% 1-RM), with a 2-min rest between sets and a 3-min rest between exercises. The laboratory temperature and relative humidity were $21.4 \pm 0.4^{\circ} \mathrm{C}$ and $64 \pm 3 \%$, respectively. Blood and saliva samples were taken during baseline, immediately after exercise, and 3 hours after exercise. Samples were refrigerated until the end of the session and centrifuged, then refrozen at $-20^{\circ} \mathrm{C}$, and stored until all sessions for each subject were completed.

Salivary cortisol concentrations were measured in duplicate by using a commercially prepared ELISA kit (Diagnostics Biochem Canada, Inc.) with modified procedures suggested by the manufacturer. A lower limit of detection for saliva cortisol was $1.0 \mathrm{ng} / \mathrm{dl}$. CK was assayed spectrophotometrically through the use of commercially available kits (PARS AZMUN CO.TEHRAN, IRAN). The CV for CK was $<4 \%$. IgA was assayed by immunodiffusion (SRID) method by use of IgA kit (The Binding Site Ltd., Birmingham, UK), The CV for IgA was $<3 \%$. 
Statistical analysis: All results are presented as mean values and standard deviation of the mean. Normality of data distribution was assessed by the KolmogorovSmirnov test. Data were analyzed using a repeated measure ANOVA and LSD post-hoc test. Statistical significance was set at $\alpha=0.05$.

\section{Results}

Results of the analysis showed that during baseline, there were no differences between exercise day (ED) and rest day (RD) for Cortisol, IgA, and CK. After exercise, Cortisol concentration in ED were significantly higher than $\mathrm{RD}(\mathrm{p}<0.05)$; however, $\operatorname{IgA}$ and $\mathrm{CK}$ responses were not significant between ED and RD. Also, 3 hours after exercise, the Cortisol concentration in ED was significantly higher than in RD $(\mathrm{p}<0.05)$; but, IgA and CK concentration 3 hours after exercise was similar in ED and RD (Table 2). In addition, there were no correlations between IgA and Cortisol responses to exercise in both days.

\section{Discussion}

This study focused on IgA, CK, and cortisol response to exercise. Consecutive aerobic and resistance exercise protocol were designed to present physiological stress similar to the preparation phase of training in most sports.

Our results shows that mean salivary concentration of IgA were unaffected by consecutive aerobic and resistance exercise. These results are in line with previous studies $[4,28,42]$. Some studies have reported decreases in IgA concentration following high-intensity exercise $[21,38]$. Their exercise protocols were undertaken during a period of overtraining [24]. However, a significant elevation in IgA concentration has also been reported after high-intensity exercise [6]. This increase seemed to result from the reduction of saliva flow rate. Previous studies have suggested that there is an intensity-dependent effect of acute exercise on IgA, i.e. strenuous exercise is suppressive and moderate exercise has no effect [23]. Therefore, the inconsistency in responses of $\operatorname{IgA}$ secretion rate to exercise may be attributed to different types of sympathetic nervous system stimulation during exercise [14]. It seems that neither aerobic exercise nor resistance exercise at the same moderate exercise intensity evokes sufficient stimulation of the sympathetic nervous system or of the hypothalamic-pituitary-adrenal axis to modify IgA transcytosis.

We found that consecutive aerobic and resistance exercise was associated with increased salivary Cortisol concentrations immediately and 3 hours after exercise. Postexercise Cortisol concentration changes seems to be affected by several mechanisms including stimulation of the sympathetic nervous system, stimulation of hypothalamic-pituitary-adrenal (HPA) secretion, increase of body temperature, changes in blood $\mathrm{pH}$, hypoxia, lactate accumulation, and mental stress [30,37]. A study reported that Cortisol concentration increases due to continued exercises. These researchers reported that physical exercise could stimulate HPA, increase body temperature, increase Cortisol secretion, and the release of Cortisol from the carrier proteins [3]. Therefore, the high concentration of salivary Cortisol accompanied by increase of saliva viscosity is the indicator of sympathetic nervous system activation [3]. The findings of this study are in line with findings of Ben-Aryeh et al. [3] and KaciubaUscilko et al. [17], who found that consecutive aerobic exercise and resistance exercise increase Cortisol concentration. Furthermore, the results of this study do not support the data given by O'Connor et al. [33] and SariSarraf et al. [37]. The equivocal nature of these observations may be due to the difference in intensity, duration, and mode of exercise, the place of exercise, and the age of the subjects in each study.

We found a lack of correlation between $\operatorname{IgA}$ and Cortisol concentrations. Specifically, we observed that IgA concentration after consecutive aerobic and resistance exercise did not change, while Cortisol concentration changed. Our findings match those of Sari-Sarraf et al. [37], reporting that during heavy and moderate exercises Cortisol secretion had no relationship with the inhibition of salivary $\operatorname{IgA}$.

Table $[$. Mean values $( \pm \mathrm{SD})$ of Cortisol, $\mathrm{CK}$ and IgA recorded pre-, post- and 3 hours post exercise during rest and exercise day in male physical education students

\begin{tabular}{|c|c|c|c|c|c|c|}
\hline \multirow[b]{2}{*}{ Variable } & \multicolumn{3}{|c|}{ Rest day } & \multicolumn{3}{|c|}{ Exercise day } \\
\hline & Pre-exercise & Post-exercise & 3h-post exercise & Pre-exercise & Post-exercise & 3h-post exercise \\
\hline Cortisol (nmol/L) & $1.26 \pm 0.3$ & $0.67 \pm 0.1$ & $0.76 \pm 0.2$ & $1.23 \pm 0.4$ & $1.43 \pm 0.2^{*}$ & $1.50 \pm 0.1 *$ \\
\hline Creatine kinase (U/L) & $200.1 \pm 12.5$ & $196.2 \pm 8.7$ & $195.1 \pm 9.1$ & $199.6 \pm 7.9$ & $203.7 \pm 10.3$ & $206.6 \pm 11.4$ \\
\hline $\operatorname{IgA}(\mathrm{mg} / \mathrm{dl})$ & $72.2 \pm 8.3$ & $67.4 \pm 6.1$ & $67.2 \pm 4.2$ & $72.4 \pm 5.4$ & $72.8 \pm 7.1$ & $72.9 \pm 6.4$ \\
\hline
\end{tabular}

Legend: IgA - Immunoglobulin A * Significantly $(\mathrm{p}<0.05)$ different from the rest day 
When the subjects work at a fixed loading, CK is released from the muscle when the loading exceeds a certain limit of muscle ability [25]. Although numerous studies $[18,32,40]$ have examined the effects of exercise on serum $\mathrm{CK}$ activity under various exercise conditions, to our knowledge no study has addressed CK release during consecutive aerobic and resistance exercise. In the present study, we found that consecutive aerobic and resistance exercise could not increase CK concentration. Previous studies reported that during exercise, muscle repeatedly contracts and uses energy substrates. When exercise intensity is within the normal range of metabolism, the muscle tissue is exercised without marked changes in membrane permeability. However, when exercise intensity exceeds this permissible range, the membrane permeability temporarily changes, resulting in $\mathrm{CK}$ release from the active muscle [25]. Volfinger et al. [41] indicated the possibility of a threshold value for CK release, with a "distance" threshold for CK release.

Totsuka et al. [25] reported that CK response depends on individual fitness level, levels that are usually different between athlete and non-athlete. Our subjects were physical education students and maybe the intensity of consecutive aerobic and resistance exercise did not exceed the ability of their muscles, thus the CK concentration in serum did not increase due to consecutive aerobic and resistance exercise.

In conclusion, we showed that 90 -min consecutive aerobic and resistance exercise led to increased salivary Cortisol, but there were no significant changes in salivary IgA and serum CK concentration. Therefore, we suggest that this type of consecutive aerobic and resistance exercise don't show a wide range of deleterious effects on the immune system. Finally, it can only be stated that this kind of consecutive aerobic and resistance exercise was well tolerated; it has demonstrated that this sub-maximal exercise did not lead to muscle damage or altered salivary IgA levels.

\section{References}

1.Ambrose C.T. (1996) Symposium on in vitro studies of the immune responses: III Biochemical agents affecting the inductive phase of the secondary antibody response initiated in vitro. Bacteriol.Rev. 30:408-417.

2.Asahi Y, T.Yoshikawa, I.Watanabe, T.Iwasak, H.Hasegawa, Y.Sato, S.Shimada, M.Nanno, Y.Matsuoka, M.Ohwaki, Y.Iwakura, Y.Suzuki, C.Aizawa, T.Sata, T.Kurata, S.Tamura (2002) Protection against influenza virus infection in polymeric Ig receptor knockout mice immunized intranasally with adjuvant-combined vaccines. J.Immunol., 168: 2930-2938.

3.Ben-Aryeh H, N.Roll, M.Lahav, R.Dlin, N.Hanne-Paparo, R.Szargel, C.Shein-Orr, D.Laufer (1989) Effect of exercise on salivary composition and cortisol in serum and saliva in man. J.Dent.Res., 68:1495-1497.
4.Bishop N.C, A.K.Blannin, P.J.Robson, N.P.Walsh, M.Gleeson (1999) The effects of carbohydrate supplementation on immune responses to a soccerspecific exercise protocol. J.Sports.Sci., 17: 787-796.

5.Bishop N.C, A.K.Blannin, N.P.Walsh, E.Armstrong, M.Rickman, M.Gleeson (2000) Carbohydrate and fluid intake affect the saliva flow rate and IgA response to cycling. Med.Sci. Sports.Exerc., 32:2046-2051.

6.Blannin A.K, P.J.Robson, N.P.Walsh, A.M.Clark, L.Glennon, M.Gleeson (1998) The effect of exercising to exhaustion at different intensities on saliva immunoglobulin A, protein and electrolyte secretion. Int.J.Sports.Med., 19:547-552.

7.Dohi K., A.M.Mastro, M.P.Miles, J.A.Bush, D.S.Grove, S.K.Leach, J.S.Volek, B.C.Nindl, J.O.Marx, L.A.Gotshalk, M.Putukian, W.J.Sebastianelli, W.J.Kraemer (2001) Lymphocyte proliferation in response to acute heavy resistance exercise in women: influence of muscle strength and total work. Eur.J.Appl.Physiol., 85:367-373.

8.Fleshner M. (2000) Exercise and neuroendocrine regulation of antibody production: protective effect of physical activity on stress-induced suppression of the specific antibody response. Int.J.Sports.Med., Supp11:14-19.

9.Fox E.L. (1973) A simple, accurate technique for predicting maximal aerobic power. J.Appl.Physiol., 35:914-916.

10. Gleeson M., W.A McDonald, D.B.Pyne, A.W.Cripps, J.L.Francis, P.A.Fricker, R.L.Clancy (1999) Salivary IgA levels and infection risk in elite swimmers. Med.Sci.Sports.Exerc., 31(1):67-73.

11. Gleeson M., D.B.Pyne (1993) Special feature for the Olympics: effects of exercise on the immune system: exercise effects on mucosal immunity. Immunol.Cell.Biol., 78:536-544.

12. Herbert T.B., S.Cohen (1993) Stress and immunity in humans: a meta-analytic review. Psychosom.Med., 55:364-379.

13. Houmard J.A., D.L.Costill, J.B.Mitchell, S.H.Park, W.J.Fink, J.M.Burns (1990). Testosterone, cortisol, and creatine kinase levels in male distance runners during reduced training. Int.J. Sports.Med., 11:41-45.

14. Hucklebridge F., A.Clow, P.Evans (1998) The relationship between salivary secretory immunoglobulin A and cortisol: neuroendocrine response to awakening and the diurnal cycle. Int.J.Psychophysiol., 31:69-76.

15. Isaacs D., A.D.Webster, H.B.Valman (1984) Immunoglobulin levels and function in pre-school children with recurrent respiratory infections. Clin.Exp.Immunol., 58:335-340.

16. Jemmott J.B., D.C.Mcclelland (1989) Secretory IgA as a measure of resistance to infections disease: Comment on Stone, Cox, Valdimarsdottir, and Neale. Behavioral.Med., 15:63-71.

17. Kaciuba-Uscilko H., B.Kruk, M.Szczpaczewsk., B.Opaszowski, E.Stupnicka, B.Bicz, K.Nazar (1992) Metabolic, body temperature and hormonal responses to repeated periods of prolonged cycle-ergometer exercise in men. J.Appl.Physiol., 64:26-31.

18. Kirwan J.P., P.M.Clarkson, J.E.Graves, P.L.Litchfield, W.C.Byrnes (1986). Levels of serum creatine kinase and myoglobin in women after two isometric exercise conditions. Eur .J.Appl.Physiol., 55:330-333.

19. Lamm M.E. (1998) Current concepts in mucosal immunity. IV. How epithelial transport of IgA antibodies relates to host defense. Am.J.Physiol., 274:G614-G617.

20. Ljungberg G., T.Ericson, B.Ekblom, D.Birkhed (1997) Saliva and marathon running. Scand.J.Med.Sci.Sports, 7:214-219. 21. Mackinnon L.T., T.W.Chick, A.Van As, T.B.Tomasi (1987) The effect of exercise on secretory and natural immunity. Adv.Exp.Med.Biol., 216A:869-876. 
22. Mackinnon L.T. (1992) Exercise and Immunology. Champaign IL: Human Kinetics.

23. Mackinnon L.T. (1996) Immunoglobulin, antibody and exercise. Exerc.Immuno.Rev., 2:1-34.

24. Mackinnon L.T., S.Hooper (1994) Mucosal (secretory) immune system responses to exercise of varying intensity and during overtraining. Int.J.Sports.Med., 15:S179-S183.

25. Totsuka M., S.Nakaji, K.Suzuki, K.Sugawara, K.Sato (2002) Break point of serum creatine kinase release after endurance exercise. J.Appl.Physiol., 93:1280-1286.

26. McDowell S.L., K.Chaloa, T.J.Housh, G.D.Tharp, G.O.Johnson (1991) The effect of exercise intensity and duration on salivary immunoglobulin A. Eur J.Appl.Physiol., 63:108-111.

27. Newham D.J., D.A.Jones, P.M.Clarkson (1987) Repeated highforce eccentric exercise: effects on muscle pain and damage. J.Appl.Physiol., 63:1381-1386.

28. Nieman D.C., D.A.Henson, O.R.Fagoaga, A.C.Utter, D.M.Vinci, J.M.Davis, S.L.Nehlsen-Cannarella (2002) Change in salivary IgA following a competitive marathon race. Int.J.Sports. Med., 23:69-75.

29. Nieman D.C., D.A.Henson, C.S.Sampson, J.L.Herring, J.M.Suttls, M.H.Conley (1995) The acute immune response to exhaustive resistance exercise. Int.J.Sports.Med., 16:322-328.

30. Nieman D.C., D.A.Henson, C.L.Dumke, R.H.Lind, L.R.Shooter, S.J.Gross (2006). Relationship between salivary IgA secretion and upper respiratory tract infection following a 160-km race. J.Sports.Med.Phys.Fitness., 46:158-162.

31. Nieman D.C., M.W.Kernodle, D.A.Henson, G.Sonnenfeld, J.M.Davis (2000) Acute immune responses to tennis drills in adolescent athletes. Res.Quartly.Exerc.Sport, 71:403-408.

32. Nosaka K., P.M.Clarkson (1992) Relationship between post-exercise plasma CK elevation and muscle mass involved in the exercise. Int.J.Sports.Med., 13:471-475.

33. O'connor P.J., W.P.Morgan., J.S.Reglin (1991) Psychobiologic effect of 3D of increased training exercise intensities on salivary cortisol. J.Strength.Cond.Res., 16:286-289.
34. Ostergaard P.A. (1977) IgA levels, bacterial carrier rate, and the development of bronchial asthma in children. Acta.Pathol. Microbiol.Scand., 85:187-195.

35. Pedersen B.K., L.Hoffman-Goetz (2000) Exercise and the immune system: regulation, integration, and adaptation. Physiol.Rev., 80:1055-1081.

36. Rahimi R., M.Ghaderi, B.Mirzaei, S.Ghaeni, H.Faraji, D.Sheikholeslami-Vatani, F.Rahmani-Nia (2010) Effects of very short rest periods on immunoglobulin A and cortisol responses to resistance exercise in men. J.Hum.Sport.Exerc., 5:146-157.

37. Sari-Sarraf V., T.Reilly, D.A.Doran, G.Atkinson (2007) The effects of single and repeated bouts of soccer-specific exercise on salivary IgA. Arch.Oral.Biol., 52:526-532.

38. Tharp G.D., M.W.Barnes (1990) Reduction of saliva immunoglobulin levels by swim training. Eur.J.Appl.Physiol., 60:61-64.

39. Tharp G.D. (1991) Basketball exercise and secretory immunoglobulin A. Eur.J.Appl.Physiol., 63: 312-314.

40. Tokuda S., A.Iiboshi, S.Otsuji (1985) Biphasic changes and characteristics in serum creatine kinase activity due to transient weight training. Jpn.J.Phys.Fitness.Sports.Med., 34:218-224.

41. Volfinger L., V.Lassourd, J.M.Michaux, J.P.Braun, P.L.Toutain (1994) Kinetic evaluation of muscle damage during exercise by calculation of amount of creatine kinase released. Am.J. Physiol.Regul.Integr.Comp.Physiol., 266:R434-R441.

42. Walsh N.P., A.K.Blannin, A.M.Clark, L.Cook, P.J.Robson, M.Gleeson (1999) The effects of high-intensity intermittent exercise on saliva IgA, total protein and alpha-amylase. J.Sports. Sci., 17:129-134.

\section{Received 04.05.2011 \\ Accepted 24.10.2011}

The authors wish to thank all the subjects for their participation and commitment to the study.

(c) University of Physical Education, Warsaw, Poland 